\title{
Degradation model of the bearings by wiener process
}

\author{
H. Louahem M'sabah*, A. Bouzaouit** \\ *20th August 1955 University, Skikda 21000, Algeria, E-mail: louahem.hanen@ gmail.com \\ **LRPCSI Laboratory, University of Skikda, 21000, Algeria, E-mail: bouzaouit@gmail.com \\ cross $^{\text {ref }}$ http://dx.doi.org/10.5755/j01.mech.22.3.13000
}

\section{Introduction}

Industrial maintenance, which aims to ensure the proper functioning of production tools, is a strategic function in companies closely linked to the incessant technological development, the appearance of new management methods, to the necessity of reducing production costs, now it has not sole purpose of repairing work tool but also to anticipate and avoid dysfunction.

Maintenance is defined as a group of activities intended to maintaining or restoring a property in a state or in the given conditions of safety function to accomplish a required function [1], the practice of maintenance is not limited only to the appreciation and traditional observation through the acceptance of a conventional maintenance, but for scientific rules or specifically non-random systems and behaviors based on simulations and modeling of diagnosis via:

- $\quad$ Essays, test, measurement and monitoring of parameters.

- Analysis of behaviours by comparison and experimentation in simulation and modelling.

- The use of digital commands and treatment with MAO.

This gave birth to a variant, mastered maintenance and efficient one through technology to finally claim towards zero failure by predictive maintenance or conditional maintenance executed following the extrapolated forecast analysis and assessment of significant parameters of the degradation of the property [2].

The aim is to act on the faulty element as close to its period of malfunctioning and also allows to follow degradation in the case of a variable life of an element all these actions are performed through the techniques of preventive conditional maintenance such as: oil analysis, thermograph and best known is the vibration analysis because it permit to detect the majority of defects that may appear in rotating machines [3].

Vibration analysis is intended to ensure the safety of the installation by avoiding significant damage by triggering an alarm when the vibration level reached judged excessive values for the functioning or integrity of the latter.

Today, with the increase in reliability of the mechanical components it is more and more difficult to estimate reliability from tests taking into account only the failure, many components are degraded before you get to failure; these degradation measures contain very precious information on the reliability of the product then it is considered that the failure occurs when the degradation reaches a certain limit [4], that's why we are interested by probability and statistical methods.
Statistical methods are now used in almost all sectors of human activity and are parts of the knowledge base of computer science, among the countless applications including in industry: the reliability of materials, analysis measurement results and planning, forecasting [5].

So we can study the degradation phenomena by stochastic processes which represent a mathematical structure used to model different phenomena; stochastic models have been many articles recent decades especially the degradation models continues, the three most frequently studied models are the following: gamma process, Poisson compound, and the Brownian movement that this article will serve to define the Wiener processes and their application in predictive maintenance.

Wiener process or the Brownian movement is a mathematical description of the random motion of a large particle immersed in the fluid and is not subject to any other interaction shocks with small molecules of the surrounding fluid it results a very irregular movement of the large particle the phenomenon was observed for the first time by the English botanist Robert Brown in 1828 [6], another interpretation was given in notes published between 1877-1880 "The Brownian movements would be in my way of looking at the phenomenon, the result of the calorific movements of molecular surrounding liquid" [7].

During the 1920 period, Norbert Wiener was the first to give a mathematical definition of Brownian motion. He studied particularly the continuity of its trajectories that are nowhere differentiable (at any time the velocity of a particle cannot be defined because its direction changes are too fast), the movement permit to describe successfully the thermodynamic behavior gas (kinetic gas theory), and the diffusion phenomenon.

\section{Methodology of work}

This study aims to follow the degradation process of bearing of a pump and allows to evaluate the operating life limit well as the evolution over time of the status change.

In the most common conditions, a bearing well calculated and well used has a $90 \%$ chance to still be in service at the end of the expected lifetime, and $10 \%$ chance of being put out of use by chipping process that constitutes somehow the natural degradation mode.

Indeed, it is recommended to program vibratory measurements at different intervals of regular time called 'age class' or periodicity of measurement, the purpose of this work is to associate a mathematical model with the results obtained during a study on the monitoring of degradation of a bearing installed on a motor pump at the Skikda refinery (Algeria). 
We execute a statistical test to the vibration measurements to summarize the information or the parameters on our sample $\left(\mu, \sigma, H_{0}, \alpha\right)$, with:

- $\quad \mu$ is the average of the model;

- $\sigma$ is a standard deviation;

- $\quad \alpha$ the degree of risk (confidence interval);

- $H_{0}$ hypothesis tested by Kolmogorov adjustment.

Then we determine this parameters of the probabilistic model and draw the two curves of the model and that of our test (model validation), finally the Wiener simulation process.

\section{Presentation of model}

The well-known process with independent increments is the Wiener process with a growing tendency; it describes increasing degradation trajectories on average. By against, it's still probable to distinguish degradation decreases over an interval that is not null [4]; we will limit ourselves to the Wiener process with linear trend.

$Y(t)$ is a Wiener process with linear trend, if $t>0$.

- $\quad Y(0)=$ almost surely.

- $\quad Y$ is a process with independent increments.

- Whatever $t>0$ and $h>0$, the increments is a normal law $N\left(m h, \sigma^{2} h\right)$.

This increment was expressed as following:

$$
\Delta Y(t)=Y(t+h)-Y(t) .
$$

The density of normal law is giving by:

$$
f(y)=\frac{1}{\sigma \sqrt{2 \pi h}} \exp \left(-\frac{(y-\mu h)^{2}}{2 h \sigma^{2}}\right) \text {, }
$$

for all $t>0, E(Y(t))=\mu t, \operatorname{Var}(Y(t))=\sigma^{2} t$ and $C V$ $C V(Y(t))=\sigma / \mu \sqrt{t}$, with $E($.$) the expectancy, \operatorname{Var}($.$) the$ variance and $C V($.) the variation coefficient.

$$
f_{T}(t)=\frac{z_{0}^{2} \sigma_{Y}}{\sigma \sqrt{2 \pi}} t^{-3 / 2} \exp \left(\frac{\left(z_{0}-\mu t\right)^{2}}{2 \sigma^{2} t}\right) \text {. }
$$

For $n$ pieces, m degradation values are observed for each piece: $Z_{i j}=Z\left(t_{i j}\right)$ where $0<t_{i 1}<\ldots . .<t_{i m_{i}}$ are the moments of observation for the piece $i$, then we consider degradation increases $\Delta Z_{i j}=Z\left(t_{i j}\right)-Z\left(t_{i(j-1)}\right), j=1 \ldots m_{i}$.

The parameters $\mu$ and $\sigma$ are obtained bymaximizing log-verisimilitude:

$$
\ln (L(\mu, \sigma))=\sum_{i=1}^{n} \sum_{j=1}^{m} \frac{1}{\sigma \sqrt{2 \pi t_{i j}}} \exp \left(\frac{\left(\Delta z_{i j}-\mu \Delta t_{i j}\right)^{2}}{2 \Delta t_{i j} \sigma^{2}}\right) .
$$

In Wiener process it is possible that the level of degradation decreases from one moment to another [8], [9]; the evolution of degradation over time depending on the normal law, this degradation is shown by the following model:

$$
Y(t+h)-Y(t)=\sigma \times N(0, h)+\mu \times h .
$$

\subsection{Statistical analysis}

This studyis achieved using a statistical software "Easy fit 5.4".This software allows to check whether the measures follow a normal distribution or not.

Below are the results of measurements of the evolution of vibration during the time. The measurements represent the effective value, also called RMS (the root mean square or the quadratic mean) of the values of each harmonic

\begin{tabular}{|c|c|c|c|}
\hline $\begin{array}{c}\text { Measure } \\
\mathrm{Nb}\end{array}$ & $\begin{array}{c}\text { Rms } \\
\text { Value (g) }\end{array}$ & $\begin{array}{c}\text { Measure } \\
\mathrm{Nb}\end{array}$ & $\begin{array}{c}\text { Rms } \\
\text { Value (g) }\end{array}$ \\
\hline 1 & 2.678 & 16 & 4.448 \\
\hline 2 & 2.743 & 17 & 4.882 \\
\hline 3 & 4.213 & 18 & 5.175 \\
\hline 4 & 3.839 & 19 & 5.887 \\
\hline 5 & 4.589 & 20 & 6.436 \\
\hline 6 & 4.029 & 21 & 6.748 \\
\hline 7 & 5.414 & 22 & 5.714 \\
\hline 8 & 7.723 & 23 & 5.712 \\
\hline 9 & 4.856 & 24 & 4.438 \\
\hline 10 & 4.453 & 25 & 4.956 \\
\hline 11 & 4.770 & 26 & 4.882 \\
\hline 12 & 4.915 & 27 & 5.971 \\
\hline 13 & 5.316 & 28 & 5.489 \\
\hline 14 & 4.667 & 29 & 6.508 \\
\hline 15 & 5.384 & 30 & 2.126 \\
\hline
\end{tabular}
vibration, followed by the different figures that represent the probability functions.

Table 1

Vibration measurement

The Kolmogorov-Smirnov adjustment permit to test the $\mathrm{H}_{0}$ hypothesis that the observed data are generated by a theoretical probability law considered a suitable model here is the result of the test:

- follows a Normal distribution;

- $\quad$ sample size $=30$;

- $\quad$ Statistics =0.12901;

- $\quad$ value of $p=0.65316$.

Table 2

Results for adjusting

\begin{tabular}{|l|l|l|l|l|l|}
\hline$\alpha$ (risk) & 0.2 & 0.1 & 0.05 & 0.02 & 0.01 \\
\hline $\begin{array}{l}\text { Critical } \\
\text { Value }\end{array}$ & 0.19032 & 0.21756 & 0.2417 & 0.27023 & 0.28987 \\
\hline Reject? & Non & Non & Non & Non & Non \\
\hline
\end{tabular}

The statistical test gave us the following results: the average is $\mu=4.9$,standard deviation is $\sigma=1.19$, we replace the values in the Eq. (2) and it will become as following:

$$
f(y)=\frac{1}{1.19 \sqrt{2 \pi h}} \exp \left(-\frac{(y-4.9 h)^{2}}{1.19^{2} 2 h}\right) \text {. }
$$


The global idea of this test is to compare the distribution of our data with theoretical distribution, the Fig. 1 is a graphical representation of the density function, the graph below in Fig. 2 represent the distribution function theoretical and measured; which serves to extract each values of the distribution function with the corresponding measurements values.

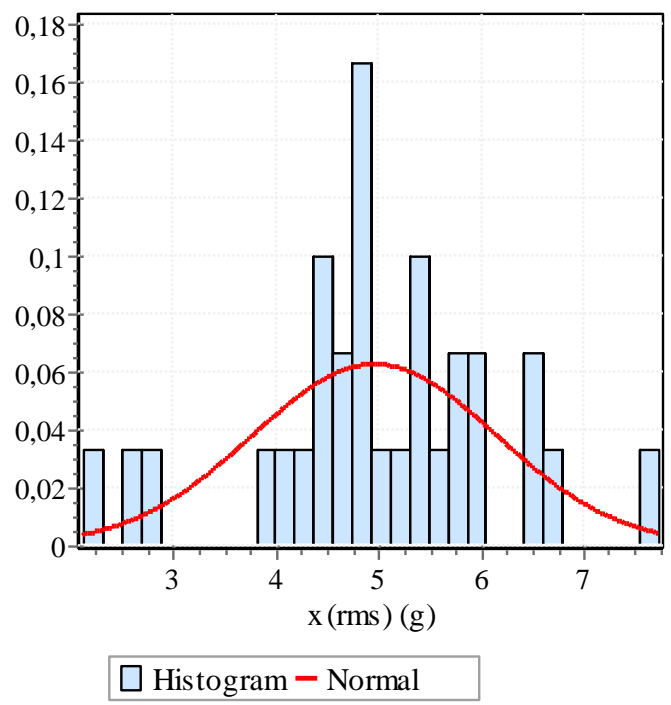

Fig. 1 Probability density function

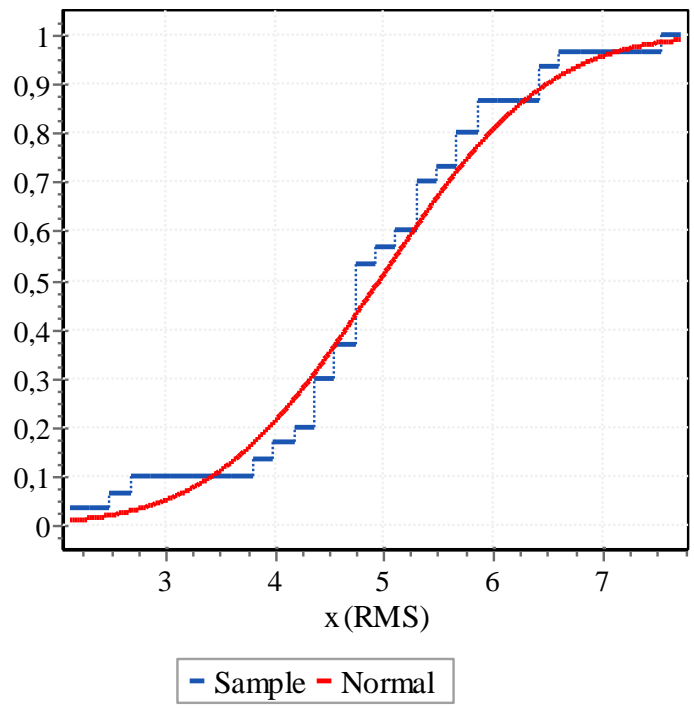

Fig. 2 Distribution function

\subsection{Model validation}

In this article we study the bearing degradation phenomenon using the Wiener process that can take in to account the negative increments wear caused by measurement errors.

Several studies show that theWiener process provides very good results from data generated by the Wiener process[4].

Degradation processes are from trajectories of stochastic process with independentgrowth, when we replace the values of $\sigma$ and $\mu$ which are respectively (the standard deviation, the average) the Wiener process characterizes increasing degradation on average this degradation follows the model interpreted in Eq. (5) so our model becomes as the following:

$Y(t+h)-Y(t)=1.19 \times N(0, h)+4.9 \times h$.

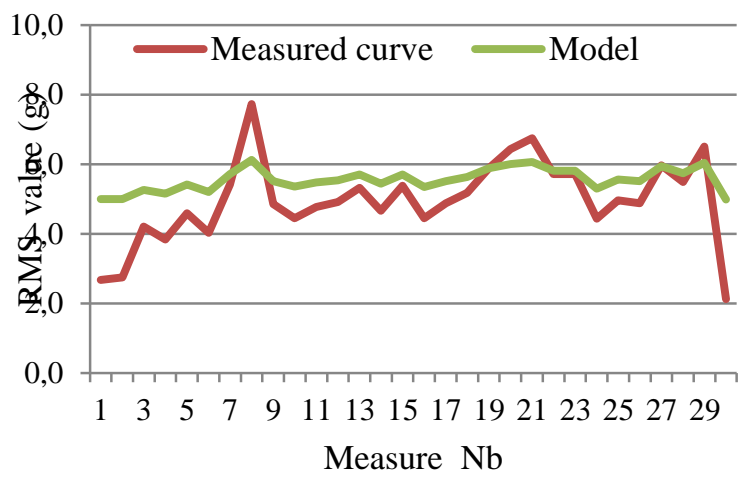

Fig.3 Presentation of the measured and modeled vibrations

\subsection{Wiener simulation}

The Wiener process helps describe increasing degradation trajectories average, this may cause a decrease in degradation between two successive times, trajectories are simulated according to a Wiener process and here are the Fig. (4) that show al trajectories.

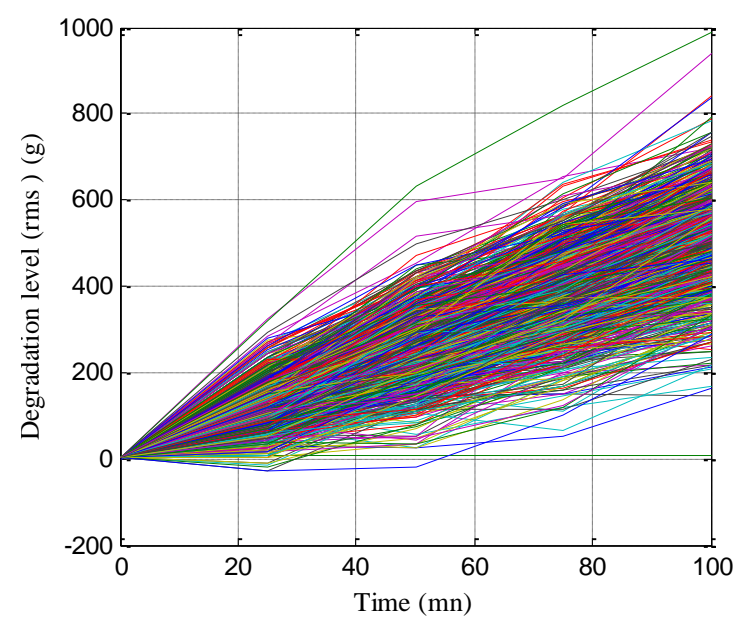

Fig. 4 Plot of 1000 trajectories according to a Wiener process parameters $\mu=4.9, \sigma=1.19$

\subsection{Interpretation of results}

The execution of the statistical test shows that vibration measurements of the degradation of the bearings follow a normal distribution $N(\sigma, \mu)$ whit a standard deviation $\sigma=1.19$ and average $\mu=4.9$, and according to adjustment of Kolmogorov observed data are accepted by the decision rule, so the hypothesis $\mathrm{H}_{0}$ was realized, the $\alpha$ values represent the values of the confidence interval extract by the test whatever $\alpha=[0.1,0.2,0.01,0.02,0.05]$, the statistical test is lower than the critical value so decision rule is accepted.

The Fig. 3 presents two curves, they are very close to each other with a correlation coefficient $k=0.971$, the curve of the probabilistic model fixed the critical level of failure of bearings because the maximum values achieved by the predictive curve is 6 , all this allows us to predict the failure of the bearings and minimizes maintenance interven- 
tions and costs by the monitoring and surveillance this benefit provides a strong motivation for the use of the model for predicting failure.

Track the progress of degradation of the bearings over time permit to model the phenomenon by the Wiener process, because degradation can be temporarily reduced by phenomena of improvement, the simulation by the Wiener process provides a good prediction.

\section{Conclusion}

This article discusses the interest of some degradation models and measurements in order to estimate the reliability of equipment, it is useful to follow the evolution of this process at different times to evaluate the state of health of the equipment and predict future evolution.

The statistical analysis methodology uses a stochastic process for the purpose of deal with the results of tests and will check the ability to model the continued deterioration of the bearings before overtaking the danger threshold because from it, bearings can be deteriorate at any time, if you want to model a phenomenon of degradation by a continuous process trajectory we chose the Brownian movement that allowed to define a model of predicted reliability therefore provide a good prediction of failures from test data, this model should also be able to help in the planning of predictive maintenance to prevent unexpected, All this provides a strong motivation for the use of the model for predicting failure.

\section{References}

1. AFNOR. Norme X60-010, 1990, Paris, Afnor.

2. François Monchy; Jean-Pierre Vernier 2010. Maintenance méthodes et organisations, Dunod, 3 edition, $536 \mathrm{p}$.

3. Djebili Omar 2013. Contribution à la maintenance prédictive par analyse vibratoire des composants mécaniques tournants: application aux butées à billes soumises à la fatigue de contact de roulements, Thèse de doctorat, Université de Reims Champagne Ardenne.

4. Baussaron, J; Barreau, M.; Gerville, L.; Guerin, F.; Schimmerling, P. 2010. Estimation de la fiabilité apartir de mesures de dégradation: Quelles méthodes utiliser? 17e congrès de maitrise des risques et de sureté de fonctionnement [5,7 octobre 2010] La Rochelle, 1-10.

5. Gilbert Saporta 2006. Probabilités et statistique analyse des données $2 \mathrm{e}$ édition révisée et augmentée.

6. Brown, R. 1828. A brief account of microscopical observations made in the months of June, July and August 1827 , on the particles contained in the pollen of plants; and on the general existence of active molecules in organic and inorganic bodies, Philosophical Magazine 4:
161-173.

7. Bertrand Duplantier 2005. Le mouvement brownien 'divers et ondoyant', Séminaire Poincaré 1, France, 155212.

8. Hersant, J.; Guérin, F.; Cloupet, S.; Germain, G. 2013. Etude d'un phénomène dégradation par usure: Phénomène physique, modélisation et analyse; QUALITA congrès, Compiègne: France, pp : 1-5.

9. Bosse, S.; Quadri, G.; Gilard, O.; Cabarbaye, A. 2012. Modèle prédictif de dégradation non linéaire dans des conditions d'environnements varies, Lambda $\mathrm{Mu} 18$ e congrès [16, 17,18 octobre 2012], 1-8.

H. Louahem M‘sabah, A. Bouzaouit

\section{DEGRADATION MODEL OF THE BEARINGS BY WIENER PROCESS}

S u m m a r y

Recently the monitoring and diagnosis of rotating machines has become an effective tool for early detection of defects and monitor changes over time.

Maintenance was considered by companies as a post of unavoidable expense for long time but today it liberates of this reputation to be regarded as a competitive factor it requires a good understanding of phenomena related to the onset and development of defects detected, the appearance of a defect at an early stage and follow their evolution shows a great industrial value, for this we execute the predictive maintenance following the extrapolated forecast of the analysis and evaluation of significant parameters of the degradation of the property, the methodology proposed in this paper aims to model the phenomenon of degradation of the bearing by via a stochastic process specially wiener process starting with the statistical test to determine the distribution of our measurements values also the parameters of the model the average $(\mu)$, standard deviation $(\sigma)$ and the degree of risk $(\alpha)$ then we draw the curve of the probabilistic model and determine the critical level of failure of bearings, it allows us to plan the operations of maintenance and improve the availability of equipment also increase the lifetime of the equipment and finally the simulation of trajectories by wiener simulation.

Keywords: predictive maintenance, vibration analysis, stochastic processes, Wiener process, modeling.

Received August 30, 2015

Accepted May 11, 2016 